\title{
An Integrated Optic Adiabatic TE/TM Mode Splitter on Silicon
}

\author{
René M. de Ridder, A. F. M. Sander, A. Driessen, and J. H. J. Fluitman
}

\begin{abstract}
A compact integrated optic fundamental TE / TM mode splitter, based on the mode-sorting characteristics of an asymmetrical adiabatic $Y$ junction of optical waveguides exhibiting shape birefringence, operating at $1550 \mathrm{~nm}$, has been designed using the discrete sine method (DSM) and the beam propagation method (BPM), realized, and tested. The structures were fabricated in a system of $\mathrm{SiO}_{2}, \mathrm{SiO}_{x} \mathrm{~N}_{y}$, and $\mathrm{Si}_{3} \mathrm{~N}_{4}$ layers, using a low-temperature plasma-enhanced chemical-vapor deposition (PECVD) process, compatible with standard silicon IC processing.
\end{abstract}

\section{INTRODUCTION}

I $\mathrm{N}$ A coherent optical communication system the polarization of the received signal should be matched to that of the signal of the local oscillator. One of the possible approaches to solving this problem is using a polarization-diversity scheme [1]. In a polarization-diversity receiver the incoming signal wave is decomposed into two orthogonal polarization components. These are processed and demodulated separately, and finally recombined.

For reasons of cost and reliability, it is advantageous if the various components making up such a receiver can be integrated on a single substrate. We believe that a silicon-based hybrid integration, going somewhat farther than the approach taken in [2], is an attractive option. Components in III-V materials (i.e., the local oscillator and the photodetectors) on the one hand and silicon oxynitride- (SiON) based passive components (e.g., waveguides, TE/TM mode converter and splitters, couplers) on the other hand can be combined on the same silicon wafer. The use of a silicon substrate, for which standard lithographic, microelectronic, and micromachining technologies are available, together with $\mathrm{SiON}$-based waveguide technology allowing the fabrication of both highcontrast and low-contrast optical waveguiding structures, is expected to facilitate the realization of an efficient and reliable fiber-chip coupling, and promises the integration with high-speed electronics. Mounting and coupling of diode lasers to a waveguide on a silicon substrate [3], as well as a passive TE/TM mode converter [4], have been demonstrated elsewhere.

Manuscript received August 11, 1992; revised May 27, 1993.

The authors are with the Mesa Research Institute, Lightwave Devices Group, University of Twente, 7500 AE Enschede, The Netherlands.

IEEE Log Number 9212907.
Another key element in such a receiver is the polarization or TE/TM mode splitter. Different operating principles for integrated optic polarization splitters are known [5]-[10]. The approaches of [5]-[8] are based on interference; therefore these devices have one or more lengths that have to be carefully adjusted to obtain the desired performance. Using the concept of mode sorting [11], critical lengths can be completely avoided. Like the authors of [9] and [10], we make use of an asymmetric $Y$ junction, as shown in Fig. 1.

If the transition from the input channel waveguide (1) to the two output guides ( 2 and 3 ) is made sufficiently gradual, it is adiabatic, and power in the fundamental mode remains in that mode throughout the transition. If the $\mathrm{Y}$ junction operates in the mode-splitting regime (see Section II), beyond the branching point the fundamental mode becomes the mode localized in the waveguide with the highest channel index, i.e., the highest effective refractive index $\left(N_{\text {eff }}\right)$. The design of the output waveguides of a TE/TM mode-splitting $\mathrm{Y}$ junction is such that one waveguide has the higher channel index for TE polarization and the other the higher channel index for TM polarization. This can be achieved by making use of the phenomenon of shape birefringence [12], which occurs in an optical waveguide having an asymmetrical refractive-index distribution, e.g., if a high-refractive-index material is interposed between the buffer and the core or between the core and the cladding. Such a thin film enlarges not only the value of the effective index of the TE and the corresponding TM mode, but also the difference between the two values, since compared to the TM mode, the modalfield distribution of the TE mode will be more concentrated in the high-index region. This results in the possibility to tune the channel index values of the waveguides 2 and 3 with respect to each other, by varying the refractive indices of the used SiON compounds and the various layer thicknesses.

The implementation of a known principle [9], [10] for the polarization splitter using a different materials system will not only affect purely technological issues, but asks for a careful and adequate design and will provide additional advantages or drawbacks. Using a plasma-enhanced chemical-vapor deposition (PECVD) SiON technology, the index of refraction can be chosen within a broad range from $1.45\left(\mathrm{SiO}_{2}\right)$ to $1.98\left(\mathrm{Si}_{3} \mathrm{~N}_{4}\right)$, giving additional freedom in the design. This materials system allows for wave- 


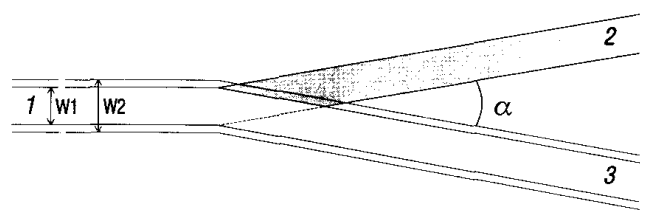

Fig. 1. Top view of an asymmetric Y junction.

guiding structures with large index contrast, resulting in thin layers, which can be efficiently coupled to, e.g., semiconductor laser diodes. A disadvantage is still the state of technology development. This is the reason why the performance reached until now is less than that of similar devices [9].

In the following the design of a TE/TM mode-splitting $Y$ junction, its simulated behavior, the realized structures, and the measurement results are presented.

\section{Design and Realization}

The design of the polarization splitting device is bound to the following constraints: realization in SiON on silicon, operating wavelength around $1550 \mathrm{~nm}$, cross talk better than $-20 \mathrm{~dB}$, while the design has to be relatively small and simple, technologically realizable, and allow a process window as large as possible. We investigate an asymmetrical Y junction as shown in Fig. 1 (top view) and Fig. 2 (cross sections of the channel waveguides). This configuration has the advantage that the sharp branching point arises as a matter of course from overlaying two smooth structures, so that it does not have to be defined directly in a critical photolighographic step.

The polarization splitter is designed using the concept of the mode conversion factor (MCF) [11], defined as

$$
\mathrm{MCF}=\frac{\left|N_{\mathrm{eff}(2)}-N_{\mathrm{eff}(3)}\right|}{\alpha \sqrt{\frac{1}{4}\left(N_{\mathrm{eff}(2)}+N_{\mathrm{eff}(3)}\right)^{2}-n_{\mathrm{eff}}^{2}}},
$$

where $N_{\text {eft(2) }}$ and $N_{\text {eff(3) }}$ are the channel indices of both output channels of the $\mathrm{Y}$ junction, $n_{\text {eff }}$ is the effective index of the surrounding (slab) region, and $\alpha$ is the splitting angle. If the absolute value of the MCF is (much) larger than 0.43 , the $\mathrm{Y}$ junction works as a mode splitter, in the way discussed in the Introduction. Consequently, the $\mathrm{Y}$ junction to be designed should have the following properties.

1) The output waveguides 2 and 3 have the largest $N_{\text {eff }}$ for TM and TE polarization, respectively.

2) For low crosstalk, the MCF value of the $Y$ junction is much larger than 0.43 for both polarizations $(\rightarrow$ small $\alpha$ ).

3) The device should be as short as possible $(\rightarrow$ large $\alpha)$.

The waveguide parameters (refractive indices, thicknesses, widths, and splitting angle, as indicated in Figs. 1 and 2) are determined in two steps. First, the layer thick- nesses and refractive indices are calculated to fulfil property 1 , taking into account the available materials and their refractive indices $\left(\mathrm{SiO}_{2}: n_{c} \approx n_{s}=1.45\right.$, SiON: $n_{g}=$ $\left.1.50-1.98, \mathrm{Si}_{3} \mathrm{~N}_{4}: n_{t} \approx 1.98\right)$. Then the less critical ridge widths and the splitting angle are determined to satisfy properties 2 and 3 . The resulting effective refractive indices of the various regions of the $\mathrm{Y}$ junction and the results of the BPM simulations are shown in Fig. 3, for both TE and TM polarization, where $\alpha$ was chosen to be $0.8^{\circ}$. A cross talk better than $-20 \mathrm{~dB}$ could be attained for any value of $\alpha$ less than $1.2^{\circ}$. The chosen value is a compromise between the shortest device length and allowance for sufficient process tolerances.

Numerical index values for both polarizations are given in Table I, where the channel indices of the waveguides 1 , 2 , and 3 were calculated using the discrete sine method (DSM) [13]. The cross talk and the excess radiation loss with respect to a straight waveguide were calculated using the BPM [14].

Since the cross talk depends on the accuracy with which the various processing steps are performed, the sensitivity to the refractive index and the thicknesses of the various layers was determined. Since in our case the structure in the SiON layer (deposited thickness $t_{4}$ ) is made by two successive etching steps (etching depths $e_{r}$ and $e_{g}$ ), the resulting thicknesses are related as $t_{2}=t_{4}-e_{r}, t_{5}=t_{4}-$ $e_{g}$, and $t_{6}=t_{4}-e_{g}-e_{r}$.

The available process window was determined in two steps. First, the cross talk was calculated as a function of each of the parameters $\left(t_{3}, t_{4}, t_{7}, e_{r}, e_{g}, n_{e}, n_{t}\right.$, and $\left.n_{c}\right)$ separately, while keeping the other parameters at their designed values. The thickness $t_{1}$ and the refractive index $n_{s}$ of the buffer layer were not varied, since $t_{1}$ was chosen so large that the evanescent field at the Si boundary was negligible, and no significant variations of the refractive index of thermal oxide were expected. It appeared that the cross talk is virtually independent of the original SiON thickness $t_{4}$ over the investigated range $1 \mu \mathrm{m} \leq t_{4} \leq 2$ $\mu \mathrm{m}$. Next, a kind of worst-case analysis was performed by varying all parameters simultaneously in such a way that their effects worked in the same direction, distributing the tolerances approximately equally over the parameters, i.e., when varying only one parameter while leaving the others at their designed values, an equal cross talk penalty was obtained. The result is shown in Fig. 4, where each label on the horizontal axis refers to a particular point in parameter space $\left(t_{3}, t_{7}, e_{r}, e_{g}, n_{g}, n_{t}, n_{c}\right)$. Label 'f' denotes the designed value and ' $a$ ' and ' $k$ ' refer to points with largest deviation from the designed value. In order to satisfy the -20 -dB crosstalk requirement, the available process window is approximately given by the range of values implied by the interval between labels ' $c$ ' and ' $i$ ' inclusive. The points in parameter space are specified in Table II.

The most critical parameters are the refractive indices, which should be controlled to within $\pm 0.25 \%$ for $\mathrm{Si}_{3} \mathrm{~N}_{4}$ and $\pm 0.3 \%$ for $\mathrm{SiON}$, and the $\mathrm{Si}_{3} \mathrm{~N}_{4}$ thickness, that has a $\pm 1.5 \%$ tolerance. The etching depth in SiON requires an 

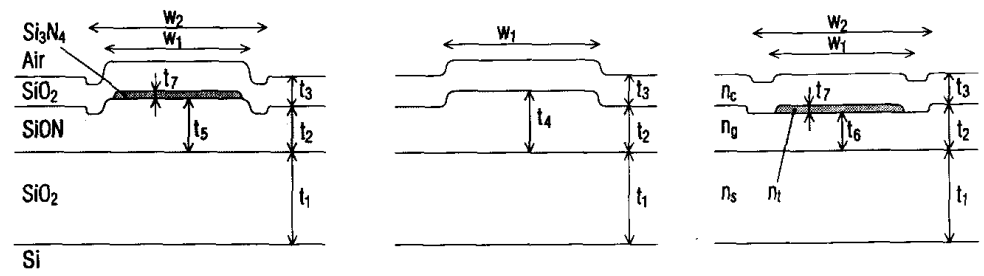

Fig. 2. Cross sections of the channel waveguides 1,2 , and 3 (left to right).
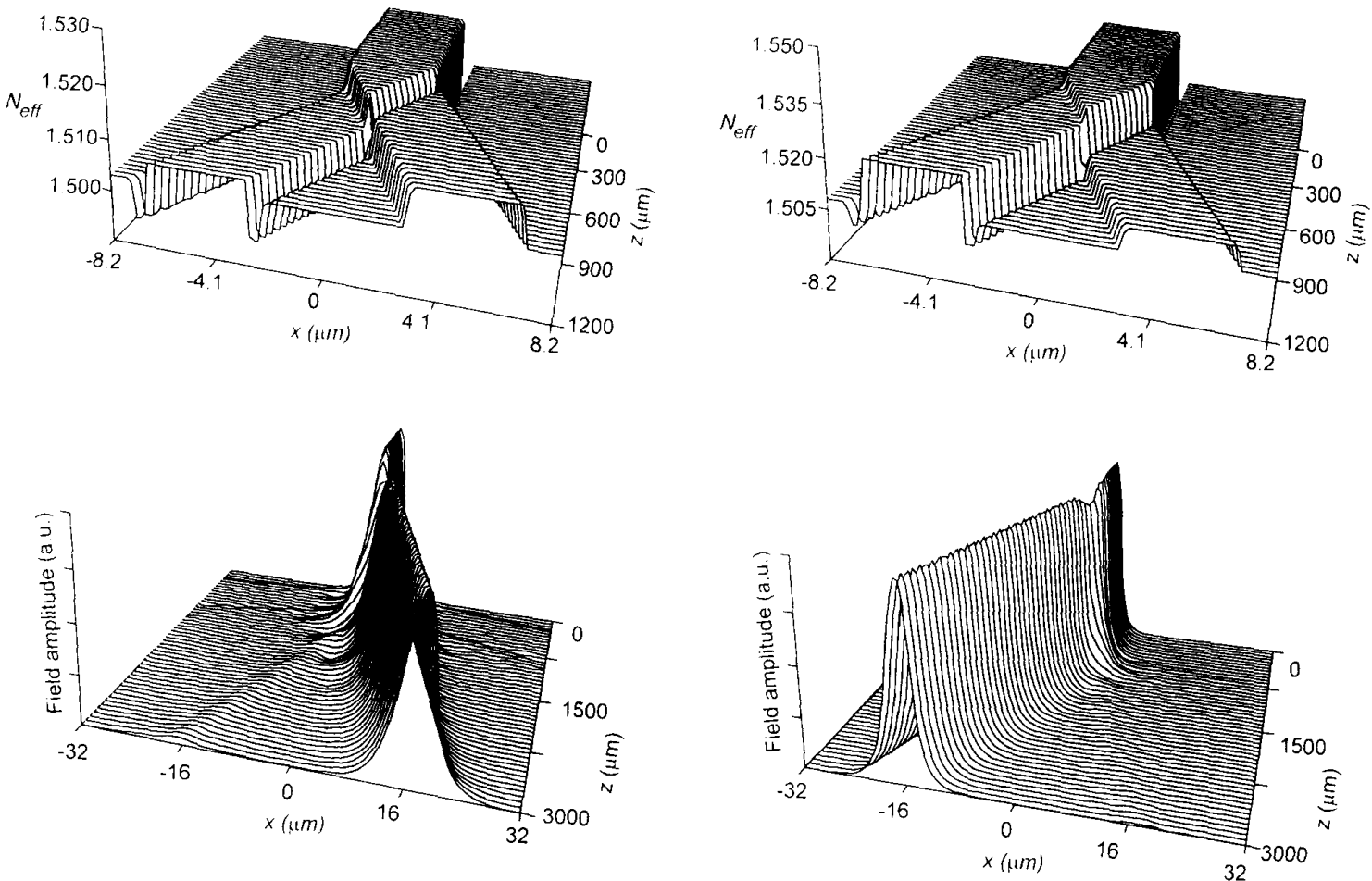

Fig. 3. Effective refractive indices of the various regions of the $Y$ junction (top), and BPM simulations of the field amplitudes (bottom), for both TE (right) and TM (left) polarization. In order to show the relevant details, the scales of the index profiles and the field profiles have been chosen differently.

TABLE I

ChanNel Indices (CALCulated Using THE DSM) OF THE WAVEGUIDES 1, 2, AND 3, THE EFFECTIVE INDEX OF THE SURROUNDING SLAB REgION $\left(n_{\text {eff }}\right)$, AND THE MODE CONVERSION FaCtor (MCF), CRoss TALK (CT), AND EXCESS Radiation LOSS WITH RESPECT TO A STRAIGHT WAVEGUIDE (LOSS), FOR BOTH TE AND TM POLARIZATION.

\begin{tabular}{cccccccc}
\hline Mode & $N_{\text {eff(1) }}$ & $N_{\text {eff(2) }}$ & $N_{\text {eff(3) }}$ & $n_{\text {eff }}$ & MCF & CT (dB) & Loss (dB) \\
\hline TE & 1.5196 & 1.5115 & 1.5150 & 1.5083 & 2.1 & -32.9 & 0.009 \\
TM & 1.5088 & 1.5071 & 1.5031 & 1.5030 & 3.6 & -25.3 & 0.006 \\
\hline
\end{tabular}

accuracy of $\pm 5 \%$. The design allows for lateral deviations (mask alignment errors, etc.) of $\pm 0.5 \mu \mathrm{m}$. The fabrication tolerances calculated above are within the range of technological possibilities.

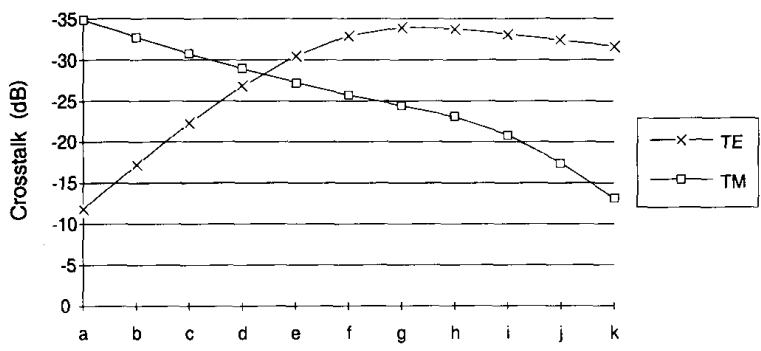

Fig. 4. Cross talk as a function of points in parameter space. Each of the horizontal-axis labels refers to a different combination of deposited layer thicknesses $\left(t_{3}, t_{7}\right)$, etching depths $\left(e_{r}, e_{g}\right)$, and refractive indices $\left(n_{g}, n_{t}\right.$, and $\left.n_{c}\right)$. Label ' $\mathrm{f}$ denotes the optimum set. (See Table II for a specification of each point ' $a$ ' through ' $f$ '.) 
TABLE II

Points in Parameter Space, as Labelled (a Through k) in Fig. 4. Each Parameter $\left(t_{3}\right.$ Through $n_{c}$ ) IS VARIED LINEARLY FROM POINT TO POINT, INCREASING OR DECREASING SO THAT THEIR EFFECTS ON the Cross Talk of the DeViations from the Designed Value (Point $f$ ) are Approximately Equal AND WORK IN THE SAME DiRECTION.

\begin{tabular}{lccccccccccc}
\hline & $\mathrm{a}$ & $\mathrm{b}$ & $\mathrm{c}$ & $\mathrm{d}$ & $\mathrm{e}$ & $\mathrm{f}$ & $\mathrm{g}$ & $\mathrm{h}$ & $\mathrm{i}$ & $\mathrm{j}$ & $\mathrm{k}$ \\
\hline$t_{3}(\mu \mathrm{m})$ & 0.280 & 0.284 & 0.288 & 0.292 & 0.296 & 0.300 & 0.304 & 0.308 & 0.312 & 0.316 & 0.320 \\
$t_{7}(\mu \mathrm{m})$ & 0.068 & 0.068 & 0.069 & 0.069 & 0.070 & 0.070 & 0.070 & 0.071 & 0.071 & 0.072 & 0.072 \\
$e_{r}(\mu \mathrm{m})$ & 0.220 & 0.216 & 0.212 & 0.208 & 0.204 & 0.200 & 0.196 & 0.192 & 0.188 & 0.184 & 0.180 \\
$e_{g}(\mu \mathrm{m})$ & 0.180 & 0.176 & 0.172 & 0.168 & 0.164 & 0.160 & 0.156 & 0.152 & 0.148 & 0.144 & 0.140 \\
$n_{g}$ & 1.558 & 1.556 & 1.555 & 1.553 & 1.552 & 1.550 & 1.548 & 1.547 & 1.545 & 1.544 & 1.542 \\
$n_{t}$ & 1.972 & 1.974 & 1.975 & 1.977 & 1.978 & 1.980 & 1.982 & 1.983 & 1.985 & 1.986 & 1.988 \\
$n_{c}$ & 1.442 & 1.444 & 1.445 & 1.447 & 1.448 & 1.450 & 1.452 & 1.453 & 1.455 & 1.456 & 1.458 \\
\hline
\end{tabular}

If $10-\mu \mathrm{m}$ separation of the output waveguides is required, the space occupied by the device will be approximately $15 \times 1000 \mu \mathrm{m}$.

The $\mathrm{SiO}_{2}$ buffer layer was thermally grown (at $1150^{\circ} \mathrm{C}$ ). The other layers were deposited using plasma-enhanced chemical-vapor deposition (PECVD) at $300^{\circ} \mathrm{C}$, which is compatible with silicon IC processing. Reactive ion etching (RIE) was used to realize the ridges and gutters. The $\mathrm{SiON}$ refractive index at various wavelengths has been related to the process parameters [15], [16]. Between processing steps, the deposited layers were characterized using a prism-coupling setup. Test structures are provided for characterizing the different slab and channel waveguide regions on the wafer.

\section{MEASUREMENTS}

The measurements on the completed devices were carried out in an end-fire coupling setup, shown in Fig. 5. The polarization could be selected using polarization filters. The surfaces of the samples were polished to make the coupling more easy to achieve. Only a small fraction of the available laser light could be coupled into the input channel of the splitter. Also, since both the coupling conditions and the propagation loss in the device were polarization dependent, no reliable data on insertion loss can be given. However, the excess loss, compared to a straight channel waveguide, could be estimated to be $-1 \mathrm{~dB}$.

The output of the channel waveguides was imaged on an infrared camera and detected with a calibrated power meter. Using the power meter, the output power from each of the output waveguides ( 2 and 3 ) was measured for both TE- and TM-polarized light coupled into the input waveguide (1). From these values, the crosstalk for TE and TM light could be calculated. The resulting values for three samples are shown in Fig. 6, exhibiting a typical cross talk of $-11 \mathrm{~dB}$ for both TE and TM polarization (best values $-17 \mathrm{~dB}$ and $-11 \mathrm{~dB}$, respectively).

\section{DISCUSSION}

Although the working principle has been qualitatively demonstrated, the experimental performance differs from the simulated performance. This has a number of causes. The realized structures deviated somewhat from the de-

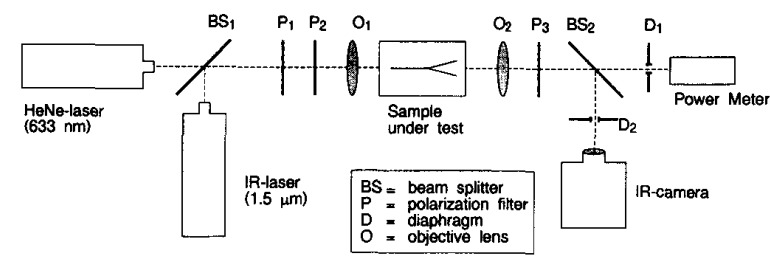

Fig. 5. Measurement setup using end-fire coupling.

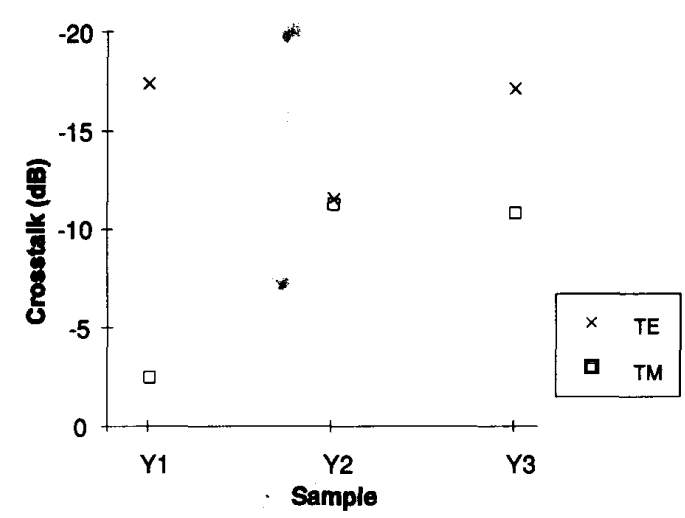

Fig. 6. Cross talk measurement on three samples Y1, Y2, and Y3.

signed ones. Fig. 7(a) shows a scanning electron microscopy (SEM) micrograph of the cross section of channel waveguide 3 . From the bottom up, the silicon substrate, the $\mathrm{SiO}_{2}$ buffer layer, the $\mathrm{SiON}$ guiding layer, the $\mathrm{Si}_{3} \mathrm{~N}_{4}$ top layer, and the $\mathrm{SiO}_{2}$ cover can be seen. As the detail shown in Fig. $7\left(\right.$ b) reveals, the $\mathrm{Si}_{3} \mathrm{~N}_{4}$ strip extends beyond the edge of the gutter, so obviously the tolerances of the lateral dimensions have been violated. The strip was approximately $1 \mu \mathrm{m}$ wider than designed, leading to channel index values deviating from the calculated ones. Besides the influence on the MCF, this could cause the realized structure to be no longer strictly monomode. It should be mentioned that only a single batch of devices was produced. It is expected that this problem will be corrected in future runs.

The results obtained with the described polarization splitter have led to an improved design, in which channel 3 is simplified: the groove in the $\operatorname{SiON}(n=1.55)$ layer 


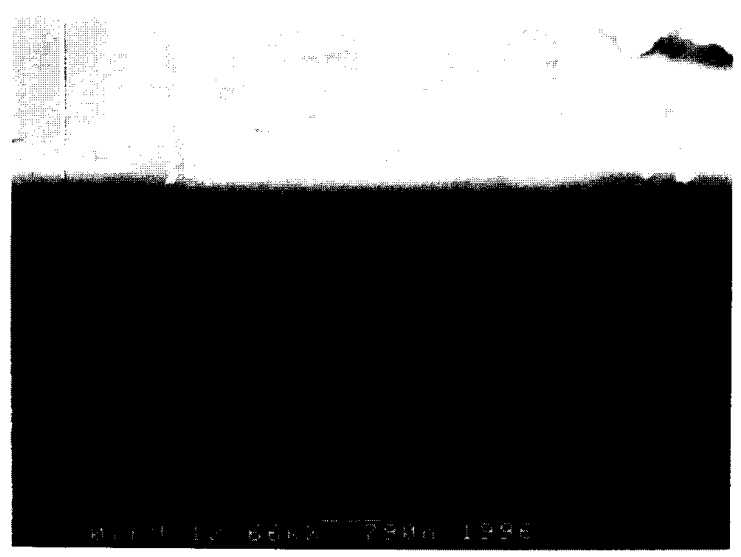

(a)

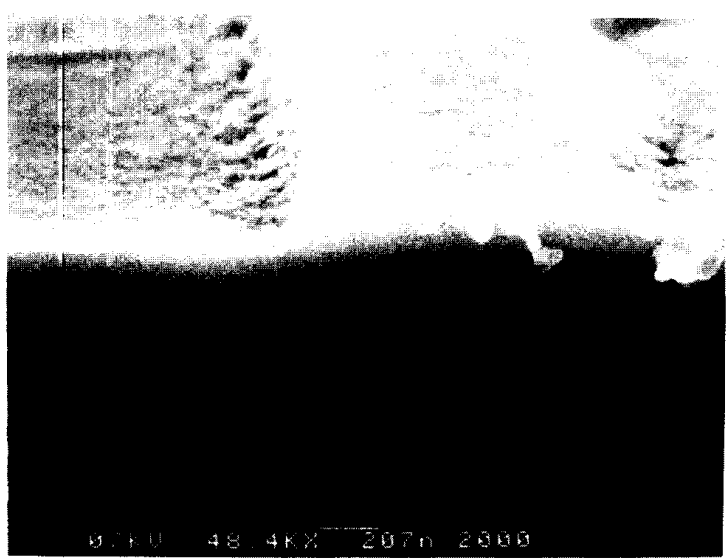

(b)

Fig. 7. SEM micrographs of a cross section of channel 3. (a) Overview of entire channel, bottom to top: $\mathrm{Si}, \mathrm{SiO}_{2}, \mathrm{SiON}, \mathrm{Si}_{3} \mathrm{~N}_{4}$, and $\mathrm{SiO}_{2}$ layers. (b) Detail of (a), showing the thin $\mathrm{Si}_{3} \mathrm{~N}_{4}$ layer to extend across the edge of the gutter in SiON, due to a photolithographic error.

disappears, so that the SiON layer thickness $t_{6}$ in the channel 3 will equal $t_{2}$. Consequently, $t_{5}$ will equal $t_{4}$. This makes the realization process essentially less complicated and thus more reliable.

The waveguides exhibited substantial attenuation, which is caused by the absorption band of the $\mathrm{SiO}_{x} \mathrm{~N}_{y}$ compounds around $1.51 \mu \mathrm{m}$, corresponding to $\mathrm{Si}-\mathrm{H}$ and $\mathrm{N}-\mathrm{H}$ bonds [17]. However, this attenuation is expected to be reduced drastically by annealing: attenuation values of 0.3 $\mathrm{dB} / \mathrm{cm}$ have been realized elsewhere after annealing PECVD SiON layers [12], [17].

\section{ConClusion}

A compact TE/TM mode-splitting $\mathrm{Y}$ junction for use in a Si-based hybrid polarization-diversity receiver has been designed for low cross talk and excess loss, and also for the allowance of a process window as broad as possible. The polarization splitter has been tested successfully. The measured cross talk of the device $(-11 \mathrm{~dB}$ for both TE and TM; best values $-17 \mathrm{~dB}$ for TE and $-11 \mathrm{~dB}$ for TM) is hopeful but still appreciably larger than the calculated values (TE: $-32.9 \mathrm{~dB}, \mathrm{TM}$ : $-25.3 \mathrm{~dB}$ ), probably due to unexpectedly large process deviations.

\section{ACKNOWLEDGMENT}

The authors thank H. M. M. Klein Koerkamp, T. H. Hoekstra, G. Krijnen, H. Albers, H. van Vossen, and G. Roelofs for their help in different stages of the project.

\section{REFERENCES}

[1] L. G. Kazovsky, "Phase- and polarization-diversity coherent optical techniques," J. Lightwave Technol., vol. 2, pp. 279-292, 1989.

[2] K. Deubel and K.-A. Steinhauser, "Microoptical heterodyne polarization diversity receiver in silicon packaging technology," Proc. ECOC' 91 (Paris), 1991, pp. 633-636.

[3] J. L. Joppe, A. J. T. de Krijger, and O. F. J. Noordman, "Hybrid integration of laser diode and monomode high contrast slab waveguide on silicon," Electron. Lett., vol. 27, pp. 162-163, 1991.

[4] Y. Shani, R. Alferness, T. Koch, U. Koren, M. Oron, B. I. Miller, and M. G. Young, "Polarization rotation in asymmetric periodic loaded rib waveguides," Appl. Phys. Lett., vol. 59, pp. 1278-1280, 1991.

[5] O. Mikami, "LiNbO 3 coupled-waveguided TE/TM mode splitter," Appl. Phys. Lett., vol. 36, pp. 491-493, 1980.

[6] A. P. Kevorkian, "A Si integrated waveguiding polarimeter," SPIE Novel Optoelectron., vol. 800, pp. 98-102, 1987.

[7] Y. Shani, C. H. Henry, R. C. Kistler, and K. J. Orlowsky, "Four-port integrated optic polarization splitter," Appl. Opt., vol. 29, pp. 337-339, 1990.

[8] A. R. Vellekoop nd M. K. Smit, "A small-size polarization splitter based on a planar optical phased array," J. Lightwave Technol., vol. 8, pp. $118-124,1990$

[9] Y. Shani, C. H. Henry, R. C. Kistler, R. F. Kazarinov, and K. J. Orlowsky, "Integrated optic adiabatic polarization splitter on silicon," Appl. Phys. Lett., vol. 56, pp. 120-121, 1990.

[10] J. J. G. M. van der Tol and J. H. Laarhuis, "A polarization splitter on $\mathrm{LiNbO}_{3}$ using only titanium diffusion," J. Lightwave Technol., vol. 9, pp. 879-886, 1991.

[11] W. K. Burns and A. F. Milton, "Mode conversion in planar-dielectric separating waveguides,” IEEE J. Quantum Electron., vol. 11, pp. 32-39, 1975.

[12] J. Aarnio, P. Heimala, M. del Giudice, and F. Bruno, "Birefringence control and dispersion characteristics of silicon oxynitride optical waveguides," Electron. Lett., vol. 27, pp. 2317-2318, 1991.

[13] H. J. W. M. Hoekstra, "An economic method for the solution of the scalar wave equation for arbitrarily shaped optical waveguides," J. Lightwave Technol., vol. 8, pp. 789-793, 1990.

[14] H. J. W. M. Hoekstra, G. J. M. Krijnen, and P. V. Lambeck, "Efficient interface conditions for the finite difference beam propagation method," J. Lightwave Technol., vol. 10, pp. 1352-1355, 1992.

[15] H. M. M. Klein Koerkamp, An integrated-optics intensity modulator based on mode coupling, Ph.D. thesis, Univ. of Twente, Enschede, The Netherlands, 1992, p. 62.

[16] W. S. Bijlsma, H. Albers, P. V. Lambeck, and Th. J. A. Popma, "PECVD $\mathrm{SiO}_{x} \mathrm{~N}_{y}$ films as planar optical waveguides," in Proc. Sensors and Actuators Symposium (Enschede, The Netherlands), 1990 , pp. 233-236.

[17] M. del Giudice, F. Bruno, R. Recca, and T. Cicinelly, "Silicon oxynitride $3 \mathrm{~dB}$ coupler for $1540 \mathrm{~nm}$ single mode applications," Proc. ECOC '91 (Paris), 1991, pp. 309-312. 


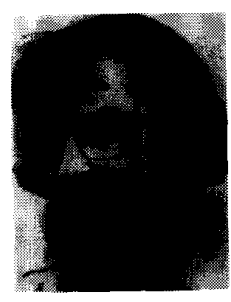

munications.
René. M. de Ridder was born April 26, 1950 in Amsterdam, The Netherlands. He studied electrical engineering at the University of Twente, Enschede, The Netherlands, where he obtained a masters degree in 1978 and a Ph.D. in 1988. Since 1981 he has been employed as a lecturer and research scientist at the University of Twente, where he is now a member of the Lightwave Devices Group in the MESA Research Institute. His current research interests include integrated-optic devices for optical com-

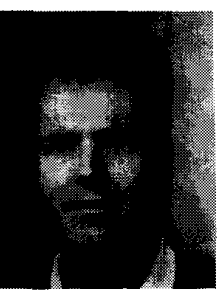

A. F. M. Sander was born on November 14 , 1964, in Weerselo, The Netherlands. In 1989 he received his M.Sc. degree in physics from the University of Twente (Enschede, The Netherlands). He completed a "technological designer" training in integrated optics in 1991. Since 1992 he has been working at the Philips Research Laboratory in Eindhoven, where his main research subject is micromechanics.

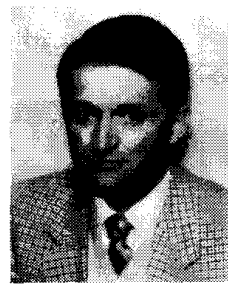

rials and devices.
Alfred Driessen (M'93) was born in Germany in 1949. In 1972 he received a degree in physics, and in 1982 the $\mathrm{Ph} . \mathrm{D}$. degree, both from the University of Amsterdam, The Netherlands.

After a 5-year period as a postdoc in the Physics Department of the Free University of Amsterdam, he became associate professor at the Lightwave Devices Group of the MESA Research Institute, University of Twente. He is mainly interested in integrated optics for telecommunication and optical nonlinear mate-

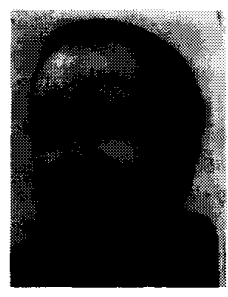

Jan H. J. Fluitman (M'80) was born on March 23, 1938, in Beverwijk, The Netherlands. He received his M.Sc. and Ph.D. degrees in physics from the University of Amsterdam in 1966 and 1970 , respectively in the field of low-temperature solid-state physics. He joined the University of Twente in 1970 . His research interest include magnetic recording, optical waveguide sensors, and micromechanics. Since 1982 he has been a full professor of transducer science and first chairman of the Sensors and Actuators Research Unit. Since 1990 he has also been Scientific Director of the MESA Research Institute, which combines microelectronics and S \& A activities.

Dr. Fluitman is a member of the MEMS steering committee, the Euro-sensors steering committee, and the World Micro Systems Technology Association. 